\title{
Physical parameters and performance values in starters and non-starters volleyball players: A brief research note
}

\author{
M.C. Marques ${ }^{1,2}$, D.A. Marinho ${ }^{1,2}$
}

1 - University of Beira Interior. Sport Sciences Department (UBI, Covilhã, Portugal)

2 - Research Centre in Sports, Health and Human Development (CIDESD, Vila Real, Portugal)

\begin{abstract}
The aim of this paper was to investigate the anthropometric and strength characteristics of elite male volleyball athletes and determines if differences exist in these characteristics between starters (S) and non-starters players (NS). A group of 22 professional male team volleyball players participated in the study and the players were categorized as $S(n=13)$ and NS $(n=9)$. Anthropometric characteristics, countermovement jump, overhead medicine ball throwing and maximal dynamic strength were evaluated in all the subjects. Significant diferences in age, hight and weight were noticed between $\mathrm{S}$ and NS. There were no significant differences between the two groups in strength and power values, except for squat performance, where $S$ were significant strong than NS. These findings provide normative data for elite male volleyball players competing in specific playing status. From a practical perspective, sport scientists and conditioning professionals should take specicif lower body strength characteristics of volleyball players into account when designing individualized training stauts specific training programmes.
\end{abstract}

Key words: anthropometrics, resistance, training, volleyball

Team Volleyball, like several other ball games, requires not only technical and tactical skills but also great deal of physical fitness (Marques, González-Badillo \& Kluka, 2006; Marques, van den Tillaar, Gabbett, Reis \& González-Badillo, 2009). During a long competitive season, typical of any European league, Team Volleyball coaches concentrate mainly on technical and tactical drills, reducing the volume of training devoted to strength and conditioning activities. This may lead therefore to unwanted changes in selected aspects of the optimal physical fitness profile.

Several studies have been undertaken to ascertain specific physical and physiological profiles of athletes in a variety of sports. For example, with respect to team sports, player profiling by position has been studied in volleyball, field hockey, basketball, netball, and soccer (Marques et al., 2009).

In volleyball, the majority of the studies have reported the characteristics of women's volleyball players or junior volleyball athletes of different However, to our best knowledge, only Marques et al. (2009) examined the anthropometric and strength characteristics of elite male volleyball athletes and determine if differences exist in these characteristics 
according to playing position. Despite the increase in professionalism, there is a paucity of research on performance characteristics of elite volleyball players.

Furthermore, usually, the potentially greater competitive responsibilities being placed on the actual starters in a sport (Kraemer et al., 2002), and the differential physiological and performance related to starters (S) or non starters (NS) status have not been clarified in prior volleyball research. In fact, few data have examined possible differences between $\mathrm{S}$ and non starters NS.

The authors were specifically interested in determining whether differences existed between $\mathrm{S}$ and NS. This study is a follow-up of a previous experiment that investigated the anthropometric and strength characteristics of elite male volleyball athletes and determines if differences exist in these characteristics according to playing position.

\section{Methods}

\section{Background}

The present research used a cross-sectional experimental design to compare the anthropometric and strength characteristics of elite male volleyball athletes according to their status: S vs. NS. This research was a longitudinal project completed over three professional competition in-seasons. All players competed in 2-3 matches per week, combined with volleyball practice sessions as well as the strength and conditioning regimen. The athletes were familiar with all of the testing and training exercises, as they had completed a pre-season training routine prior to the initiation of the current in-season study. Consequently, the athletes were in good overall physical condition and were adequately familiarized with all experimental procedures.

\section{Subjects}

A group of 22 professional male team volleyball players (mean \pm SD age: $26.2 \pm 3.3$ years) participated in the study. Players were categorized as $\mathrm{S}(n=13)$ and NS $(n=9)$. The subjects were required to sign an information and informed consent form prior to the study that had been approved by the Institutional Review Committee Board of the local Committee for Medical Research Ethics and current Portuguese law and regulations, and carried out according to the Helsinki Declaration.

\section{Training History}

Apart from standard technical and tactical practice sessions (3-4 hours per day) and competitions, the subjects completed a resistance-training regimen (RT) that included upper and lower body exercises targeting strength and power. Briefly, the resistance program was performed twice per week during the 12 -week in-season, with each session 
lasting approximately 60 minutes. The principal resistance exercises were the bench press and parallel back squat. Subjects performed 3 sets of 3-6 repetitions for each exercise in the range of $50-85 \%$ of their predetermined 4 -repetition maximum, with the loads adjusted throughout the season. Subjects also completed upper and lower body power exercises (loaded and unloaded vertical jumping activities and medicine ball throwing).

\section{Testing Procedures}

A detailed description of the muscle power testing procedures can be found elsewhere (Marques \& González-Badillo, 2006). Testing was carried out over 1 week, at the completion of the first preparatory phase (4-weeks). During this initial preparatory phase the subjects performed 3 sets of 8-10 repetitions using moderate loads for the bench press and squat exercises, plus unloaded jumping drills, and medicinal ball throws. Consequently, all the subjects were familiarized with the testing procedures and exercises, which they had been performing as part of their training routine. Maximal strength and explosive tests were separated by a 3 -day period. These were tests that could be rapidly administered, and were highly specific to team volleyball.

Anthropometric measurements: The anthropometric variables of height $(\mathrm{m})$ and body mass $(\mathrm{kg})$ were measured in each subject (Table 1 ). Height and body measurements were made on a levelled platform scale (Año Sayol, Barcelona, Spain) with an accuracy of $0.01 \mathrm{~kg}$ and $0.001 \mathrm{~m}$, respectively. Arm span measurement was performed by two trained observers. One observer took all span measurements of all subjects with help from the other observer who made sure that the arms were straight during measurements. Arm span was measured ( $m$ ) with a calliper steel tape from tips of needle fingers of maximally outstretched hands with subject standing and facing the wall.

Table 1. Selected characteristics of the subjects

\begin{tabular}{cc}
\hline Tests $(\mathrm{S}+\mathrm{NS})$ & $\begin{array}{c}\text { Anthropometric characteristics } \\
\text { Variables }\end{array}$ \\
\hline Age S -13 & $27.4 \pm 2.2$ years * \\
Age NS -9 & $24.5 . \pm 4.0$ years \\
Height S -13 & $1.93 \pm 0.5 \mathrm{~m}$ \\
Height NS -9 & $1.91 \pm 0.6 \mathrm{~m}$ \\
Weigth -13 & $92.6 \pm 6.8 \mathrm{~kg}$ \\
Weigth NS - 9 & $89.4 \pm 7.0 \mathrm{~kg}$ \\
\hline
\end{tabular}

* Significant difference

Countermovement jump: Countermovement jump (CMJ) height was measured using a trigonometric carpet (Ergo jump Digitime 1000, Digest Finland). Subjects began from a standing position, performed a crouching action followed immediately by a jump for maximal height. Each subject completed three attempts with two minutes of rest allowed between trials. The hands were on the hips during the whole jump. The average of the two 


\section{0 | Physical parameters and performance values in volleyball players}

best trials was used for analysis. The CMJ had an intraclass correlation coefficient (ICC) of 0.98 .

Overhead Medicine Ball Throw: An overhead medicine ball throw was used to evaluate the upper body ability to generate muscular actions at a high rate of speed. While standing, subjects held a three $\mathrm{kg}$ medicine ball in both hands in front of the body with arms relaxed. The athletes were instructed to throw the ball over their heads as far as possible. A countermovement was allowed during the action. Five trials were performed with a oneminute rest between each trial. An average of the best four throws was subsequently used for analysis. The distance of the throw was recorded to the closest one-quarter inch. The ball throwing distance (BTd) showed an ICC of 0.96 .

Maximal Dynamic Strength: The maximal strength tests for the upper and lower muscles were carried out using a 4 -repetition maximum bench press ( $4 \mathrm{RM}-\mathrm{BP}$ ) and a ${ }_{4} \mathrm{RM}$ parallel squat (4RM-PS). The ${ }_{4} R M-B P$ test was conducted on a standard bench and required the subject to perform an eccentric-concentric action. Beginning with the arms fully extended, the athletes lowered the bar towards the chest reaching $90^{\circ}$ abduction of the shoulder joint and $90^{\circ}$ flexion of the elbow before returning to the start position. Repetitions performed incorrectly were not included in the count. The protocol began with $20 \mathrm{~kg}$ and increased 10, 5 , and $2.5 \mathrm{~kg}$ during subsequent sets until four complete repetitions could not be performed. All subjects performed 5-6 reps for each warm-up set. The rest time between sets was 3 minutes.

For the 4 RM-PS, subjects started with $50 \mathrm{~kg}$, performing on command a series of four parallel squats. Subsequently, the weight was increased by $10 \mathrm{~kg}$ and $5 \mathrm{~kg}$ increments until the subject was unable to reach full extension of the legs. The high-bar parallel squat (4RMPS) consisted of the barbell being placed on the superior aspect of the trapezius, while the feet were placed shoulder-width apart and angled laterally approximately $15-30^{\circ}$. Parallel squat depth for this study consisted of the inguinal fold at the same level as the superior aspect of the knee. Twenty-minute rest intervals separated the ${ }_{4} R M-B P$ and ${ }_{4} R M-P S$ tests. The 4 RM-BP showed an ICC of 0.94. The 4RM-PS reported an ICC of 0.95 .

\section{Statistical Analyses}

Statistical analysis followed the most important descriptive statistics, such as mean and SD. A t-test was used to determine significant differences among playing positions in physical and performance characteristics. The level of significance was set at $p \leq 0.05$ and all data are expressed as mean \pm SD.

\section{Discussion and findings}

Tables 1 and 2 show the anthropometric, strength and power results for $\mathrm{S}$ and NS players. Significant diferences in age were noticed between $S$ and NS $(p=004)$. In addition, $S$ tend to be heavier and taller compared to $\mathrm{S}$. 
Of the grate interest of this research was that there were no significant differences between the 2 groups in strength and power values, except for squat performance. Here, $\mathrm{S}$ were significant strong than NS $(p=0.018)$.

These findings provide normative data for elite male volleyball players competing in specific playing status. From a practical perspective, sport scientists and conditioning professionals should take specicif lower body strength characteristics of volleyball players into account when designing individualized training stauts specific training programmes.

Table 2. Performance characteristics of individual playing positions in professional male Volleyball players

\begin{tabular}{ccc}
\hline Tests (S+NS) & Testing Variables & $p$ value \\
\hline CMJ S - 13 & $45.5 \pm 4.2 \mathrm{~cm}$ & $\mathrm{NS}$ \\
CMJ NS -9 & $44 \cdot 7 . \pm 3.4 \mathrm{~cm}$ & \\
BTd S - 13 & $11.9 \pm 1.2 \mathrm{~m}$ & $\mathrm{NS}$ \\
BTdS NS - 9 & $11.78 \pm 1.16 \mathrm{~m}$ & \\
4RM-BP S - 13 & $90 \pm 13 \mathrm{~kg}$ & $\mathrm{NS}$ \\
4RM-BP NS - 9 & $83 \pm 13 \mathrm{~kg}$ & \\
4RM-PS S - 13 & $143 \pm 19 \mathrm{~kg}$ & 0.018 \\
4RM-PS NS - 9 & $129 \pm 21 \mathrm{~kg}$ & \\
\hline
\end{tabular}

NS: non significant differences

\section{References}

Kraemer, W.J., Koziris, L., Ratamess, N.A., Häkkinen, K., Triplett-Mcbride, N.T., Fry, A.C., et al. (2002). Detraining produces minimal changes in physical performance and hormonal variables in recreationally strength-trained men. Journal of Strength and Conditioning Research, 16, 373-382.

Marques, M.C., \& González-Badillo, J.J. (2006). In-season resistance training and detraining in professional team handball players. Journal of Strength and Conditioning Research, 20, 563-571.

Marques, M.C., González-Badillo, J.J., \& Kluka, D. (2006). In-season strength training male professional volleyball athletes. Strength and Conditioning Journal, 28(6), 2-12.

Marques, M.C., van den Tillaar, R., Gabbett, T.J., Reis, V.M., \& González-Badillo, J.J. (2009). Physical fitness qualities of professional volleyball players: Determination of positional differences. Journal of Strength and Conditioning Research, in press. 